Justice sociale, non-discrimination, non-nuisance et nécessité de considérer les conditions nécessaires au bon développement de l'enfant

\title{
La procréation médicalement assistée
}

Commission nationale d'éthique dans le domaine de la médecine humaine (CNE-NEK)
Correspondance:

CNE-NEK

c/o Office fédéral de la

santé publique

CH-3003 Berne

Tél. 0313240236

nek-cne[at]bag.admin.ch
La Commission nationale d'éthique dans le domaine de la médecine humaine (CNE-NEK) a publié sa prise de position intitulée «La procréation médicalement assistée: Considérations éthiques et propositions pour l'avenir» ( $\left.n^{\circ} 22 / 2013\right)$. En adoptant une perspective générale, la commission y traite les questions posées par la procréation médicalement assisté (PMA) sur le plan de l'éthique - ceci du point de vue médical et du point de vue sociétal -, ainsi que sur le plan du droit. La commission soumet à un examen critique les valeurs normatives (dignité humaine, famille, bien de l'enfant, liberté et «nature» ou «naturel») sur lesquelles reposent les dispositions légales actuelles, tout en inscrivant ses réflexions dans le non mariés, alors que la majorité de la commission est favorable à l'autorisation du don de sperme pour les personnes seules et les couples de même sexe. La CNE souhaite aussi que soit levée l'interdiction du don d'ovule et d'embryon.

La commission aborde également la question de la maternité de substitution et le statut juridique des enfants nés à l'étranger grâce à une mère porteuse: ces enfants et leurs parents sont confrontés à de graves difficultés lors de leur entrée en Suisse, notamment du point de vue de l'état civil. Si la majorité de la commission estime que la maternité de substitution peut être admissible sur le principe, elle émet des doutes quant à la possibilité d'un encadrement

\section{Elle recommande à la majorité l'autorisation des tests préimplantatoires, au nom de la solidarité envers les couples et familles concernés par la transmission de maladies héréditaires graves.}

contexte social actuel. Elle formule enfin 13 recommandations.

Dans les trois premières recommandations, la commission prend position sur les implications juridiques et éthiques de plusieurs types de tests préimplantatoires - à visée diagnostique, de dépistage et de typage tissulaire pour sélectionner un donneur compatible - comme manières de prévenir la survenue d'un tort prévisible. Elle en recommande à la majorité l'autorisation, au nom de la solidarité envers les couples et familles concernés, ou déjà touchés, par la transmission de maladies héréditaires graves. Il y va aussi à son avis de la cohérence du système législatif (dont on rappelle qu'il autorise le diagnostic prénatal - menant à des interruptions de grossesse plus tardives)

Les trois recommandations suivantes s'expriment sur les modalités et les formes de don de gamètes et d'embryons. Au nom de l'égalité de traitement et du principe de non-discrimination, la commission recommande unanimement l'autorisation du don de sperme pour les couples hétérosexuels acceptable assurant la protection adéquate de toutes les personnes concernées, notamment en considération des dangers liés aux intérêts économiques que cette pratique génère.

Enfin la CNE-NEK attire l'attention sur l'importance que les dispositions légales tiennent compte des bonnes pratiques médicales. À ce titre elle plaide

\section{La CNE souhaite que soit levée I'interdiction du don d'ovule et d'embryon.}

pour la levée de toute détermination d'un nombre maximal d'embryons pouvant être développés dans le cadre de la PMA et elle recommande à l'unanimité la création d'un registre des enfants nés par PMA.

La prise de position est disponible sous www. nek-cne.ch $\rightarrow$ Publications. 